\title{
Estudio Histológico del Tracto Digestivo del Neón Cardenal Paracheirodon axelrodi (Characidae)
}

\author{
Histological Study of Digestive Tract of the Neón Cardenal Paracheirodon axelrodi (Characidae)
}

Gómez-Ramírez, E; Obando, M. J; Tovar, M. O; Caldas, M. L. \& Hurtado, H.

GÓMEZ-RAMÍREZ, E.; OBANDO, M. J.; TOVAR, M. O.; CALDAS, M. L. \& HURTADO, H. Estudio histológico del tracto digestivo del Neón Cardenal Paracheirodon axelrodi (Characidae). Int. J. Morphol., 29(3):782-786, 2011.

RESUMEN: Paracheirodon axelrodi es el pez ornamental que más se exporta en Colombia. Sin embargo, se desconocen varios aspectos de su biología básica. Se estudió la histología del tracto digestivo. Se sacrificaron 15 ejemplares (MS 222, 0,5 g/L), fijados en formaldehído al $4 \%$ y decalcificados con ácido nítrico $7 \%$. Se siguió el procedimiento para H\&E. El tracto digestivo de P. axelrodi presentó cuatro capas constitutivas: mucosa, submucosa, muscular y serosa. La mucosa de la boca presentó un epitelio escamoso estratificado no queratinizado con células caliciformes y sin botones gustativos, una submucosa de tejido conectivo laxo y una capa de músculo estriado esquelético. Los dientes son cónicos y abundantes. La capa serosa a lo largo del tracto digestivo es delgada compuesta de tejido conectivo laxo. La capa mucosa, submucosa y muscular del esófago posee la misma conformación tisular de la boca, sin embargo, la capa muscular presenta dos orientaciones. La mucosa del estómago está compuesta por epitelio cilíndrico simple con glándulas gástricas, submucosa de tejido conectivo laxo y una capa de músculo liso en dos orientaciones. Las capas del intestino exhibieron una composición tisular similar a la del estómago, sin glándulas gástricas. La mucosa presentó células caliciformes especialmente hacia la región posterior y un aumento en la longitud de los pliegues intestinales. La conformación tisular y morfológica del tracto digestivo de $P$. axelrodi lo ubica como un pez de hábitos alimenticios carnívoros pero de pequeñas presas.

PALABRAS CLAVE: Paracheirodon axelrodi; Tracto digestivo; Histología; Morfología.

\section{INTRODUCCIÓN}

El Neón cardenal Paracheirodon axelrodi es la principal especie de pez ornamental exportada desde Colombia (Mancera \& Álvarez 2008). Este pez es predador, alimentándose principalmente de micro-crustáceos como cladóceros, dafnias y larvas de quironómidos (Walker, 2004). En los últimos años han cobrado fuerza los estudios histológicos de tracto digestivo relacionando la morfología con la ecología y evolución de las especies ícticas (Albrecht et al., 2001), debido a que los teleósteos poseen características morfológicas y fisiológicas particulares en la configuración del tubo digestivo (Hepher, 1993). Se desconocen muchos aspectos de la biología de $P$. axelrodi (Walker). Por lo cual, se planteó el estudio histológico de su tracto digestivo.

\section{MATERIAL Y MÉTODO}

El procedimiento se llevó a cabo teniendo en cuen- ta lineamientos éticos (AVMA, 2001). Se trabajó con 15 ejemplares de $P$. axelrodi de longitud total $2,7+/-0,19 \mathrm{~cm}$. Se sacrificaron con MS-222 (0,5 g/L) (Tovar et al., 2009). Se fijaron en formaldehído al $4 \%$, $(\mathrm{pH} 7,2-7,3)$, durante cinco días a $4{ }^{\circ} \mathrm{C}$ (Jaramillo et al., 2009). Se decalcificó con ácido nítrico al $7 \%$ a $18^{\circ} \mathrm{C}$ durante siete días, se realizaron cinco lavados con agua corriente de media hora cada uno; y se dejaron nuevamente en formaldehído durante 24 horas. Posteriormente los individuos fueron sometidos a un proceso de deshidratación e inclusión en parafina (Jaramillo et al.). Se realizaron cortes transversales semiseriados de $5 \mu \mathrm{m}$ de espesor en un micrótomo rotatorio (MICROM HM 340E). Los cortes se tiñeron con la técnica de H\&E (Jaramillo et al.). Para el estudio histológico se tuvo en cuenta la presencia de las diferentes capas características del sistema digestivo; mucosa, submucosa, muscular y serosa, así como también los tejidos que conforman cada una de las capas en cada uno de los órganos evaluados (Olaya et al., 2007). 


\section{RESULTADOS}

Descripción macroscópica: La boca es terminal y relativamente grande (Fig. 1). En las mandíbulas (superior e inferior) se puede observar algunos dientes de forma cónica. Branquioespinas pocas y cortas. El esófago es corto y musculoso. El estómago es grande y en forma de "J" musculoso. Se distingue la región cardíaca y la pilórica. No se observan ciegos pilóricos. El intestino es corto con pocas asas y no se observan diferencias entre regiones.

Descripción microscópica: Las mandíbulas (superior e inferior) están compuestas por cartílago hialino (Fig. 1) y en ellas se puede observar algunos dientes constituidos por vasodentina, la pulpa, la cápsula enameloide y la unión ósea (Fig. 3).
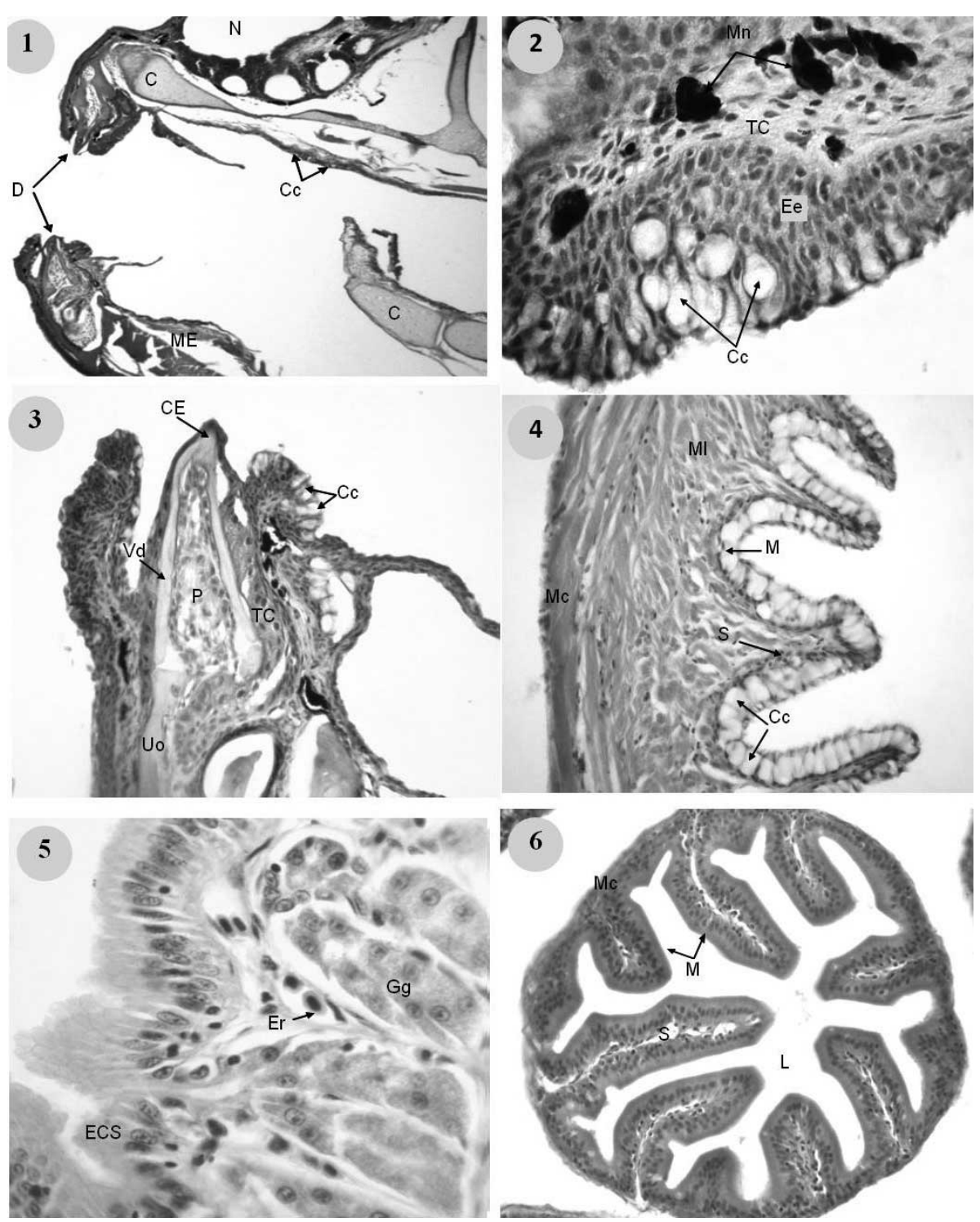

En general, los diferentes órganos que componen el tracto digestivo presentaron cuatro capas constitutivas: mucosa, submucosa, muscular y serosa.

Mucosa: en la cavidad bucal y el esófago tiene epitelio plano estratificado no queratinizado con abundantes células caliciformes (Figs. 2 y 4). En el estómago está compuesta por epitelio cilíndrico simple, con glándulas gástricas tubulares que se caracterizan por presentar células secretoras cuyos núcleos se localizan centralmente (Fig. 5). Hay mayor abundancia de glándulas gástricas en la región cardíaca. En el intestino está compuesta de epitelio cilíndrico simple (Fig. 6), sin glándulas gástricas, con células caliciformes, principalmente hacia la parte posterior.

Fig. 1. Cavidad bucal de P. axelrodi. Células caliciformes $(\mathrm{Cc})$, Cartílago hialino (C), Diente (D), Narina (N), Músculo estriado esquelético (ME). Hematoxilina y Eosina (H\&E). 20X.

Fig. 2. Detalle del piso de la cavidad bucal de $P$. axelrodi. Células caliciformes $(\mathrm{Cc})$, Tejido conectivo laxo (TC), Epitélio escamoso estratificado (Ee), Melanoforos (Mn). (H\&E). 800X.

Fig. 3. Diente de P. axelrodi. Células caliciformes $(\mathrm{Cc})$, Cápsula enameloide (CE), Vasodentina (Vd), Pulpa (P), Tejido conectivo (TC), Unión ósea (Uo). (H\&E). 320X.

Fig. 4. Esófago de P. axelrodi. Células caliciformes $(\mathrm{Cc})$, Mucosa (M), Submucosa (S), Músculo longitudinal $(\mathrm{Ml})$ y músculo circular (Mc). (H\&E). 80X.

Fig. 5. Estómago de $P$. axelrodi. Epitélio cilíndrico simple (ECS), Glándulas gástricas (Gg), Eritrócitos (Er). (H\&E). 800X.

Fig. 6. Intestino de $P$. axelrodi. $\mathrm{Mu}-$ cosa (M), Submucosa (S), Músculo liso circular (Mc), Lúmen (L). (H\&E). 80X. 
Submucosa. Tanto en la cavidad oral, como en el esófago, el estómago y el intestino presentan una submucosa de tejido conectivo laxo (Figs. 2, 4 y 6). En la primera se encuentran numerosos melanocitos (Fig. 2).

Muscular. En la cavidad bucal y el esófago esta capa es de músculo estriado esquelético con varias orientaciones para el caso de la boca (Fig. 1) y con dos orientaciones en el esófago: circular interna y longitudinal externa (Fig. 4). En el estómago es una capa de músculo liso relativamente gruesa con dos orientaciones: una circular interna y una longitudinal externa. En el intestino es de músculo liso, dos orientaciones circular interna y una longitudinal externa, siendo más delgada que en las otras regiones del tubo digestivo (Fig. 6).

Serosa. En todos los órganos la serosa está constituida de tejido conectivo laxo muy delgado.

\section{DISCUSIÓN}

El tracto digestivo de $P$. axelrodi se encuentra organizado de manera similar a la presentada por otros teleósteos que poseen estómago verdadero (Steffens, 1987; Caceci et al., 1997; Albrecht et al.; Muñoz et al., 2006; Olaya et al.).

$P$. axelrodi posee únicamente dientes de forma cónica, similar a $G$. morhua (Morrison, 1987) a diferencia de $P$. brachypomus que presenta dientes molariformes y cónicos, y de los villiformes presentados por los Silúridos (Beltrán \& Beltrán 1973; Vásquez, 2004; Olaya et al.). La disposición bucal y los dientes cónicos de $P$. axelrodi hace suponer que es un pez de hábitos carnívoros que traga las presas enteras, pero de tamaño pequeño. Así mismo, estas presas deben ser de consistencia blanda por la abundancia de cartílago hialino en la mandíbula, diferente a lo presentado por alevinos de $P$. brachypomus (mandíbula osificada) pues al tratarse de una especie omnívora-frugívora debe romper frutos y bayas (Muñoz et al.). Las características morfológicas encontradas en la boca de $P$. axelrodi coincide con los ítems alimenticios reportados por Walker. Las branquias de $P$. axelrodi al igual que las de $P$. brachypomus (Muñoz et al.), Leporinus friderichi y Leporinus taeniofasciatus (Albrecht et al.) no parecen cumplir funciones alimenticias debido a su forma y al bajo número de branquioespinas.

Aunque en otras especies es común encontrar botones gustativos en la boca, faringe y la porción craneal del esófago, en $P$. axelrodi no fueron identificados, una posible explicación para la ausencia de estas estructuras es lo reportado por Tovar et al., el cual evidenció un sistema visual desarrollado en esta especie, lo que indicaría que P. axelrodi depende en gran medida de la visión para capturar sus presas y no del gusto para detectarlas. La mucosa del esófago de $P$. axelrodi es similar a la de otros teleósteos (Anderson \& Mitchum, 1974; Morrison; Caceci et al.; Albrecht et al.; Santana \& Verdugo, 2003; Muñoz et al.; Olaya et al.). Esta conformación tisular de la mucosa del esófago le sirve para evitar el daño al ingerir presas vivas, o alimentos de consistencia dura (Olaya et al.). Para facilitar la ingestión, las células caliciformes cumplen esencialmente dos funciones: secretar mucopolisacaridos para lubricación (Amin et al., 1992; Olaya et al.) y producir co-factores enzimáticos o enzimas hidrolíticas (Albrecht et al.). La submucosa del esófago es similar a la de P. blochii, P. brachypomus y P. pictus (Santana \& Verdugo; Muñoz et al.; Olaya et al.) pero difiere a lo reportado por Morrison para G. morhua (tejido conectivo denso, mayor fortaleza y rigidez). Se sugiere que esta conformación en $P$. axelrodi permite mayor expansión del órgano y al estar relacionado con una capa muscular que presenta dos orientaciones le confiere mayor capacidad de distensionamiento (Olaya et al.). Al poseer un músculo estriado esquelético, permite un movimiento controlado el cual es importante para evitar la ingesta excesiva de agua y regurgitar el alimento no deseado (Morrison; Vásquez; Muñoz et al.; Olaya et al.). El epitelio del estómago es similar al de varios teleósteos (Anderson \& Mitchum; Morrison; Caceci et al.; Albrecht et al.; Santana \& Verdugo; Muñoz et al.; Olaya et al.), y posiblemente cumpliría funciones de secreción de pepsina y acido clorhídrico (Collette et al., 2000), aunque no podría descartarse la presencia de amilasas, lipasas, maltasas y quitinasas. Esta última se ha reportado en las familias Percichthydae y Scombridae; grupos que consumen crustáceos y zooplancton (Saga \& Roman, 1995), dieta similar a la de $P$. axelrodi (Walker). La capa muscular del estómago y del intestino está constituida por músculo liso con dos orientaciones aunque de mayor espesor en el estómago, lo cual permite que los movimientos peristálticos se efectúen en varias direcciones y que la acción enzimática sea más eficiente durante el proceso de digestión (Olaya et al.). Dicho proceso asegura el desdoblamiento de gran parte de los alimentos antes de llegar al intestino, pues al tratarse de un pez con estómago verdadero, la mayor parte de la absorción ocurre a lo largo del intestino (Velásquez; Olaya et al.) y para este trabajo se encontró que este es corto y con muchos pliegues.

La capa muscular del intestino fue la más delgada de todos los órganos estudiados, similar a lo presentado por $P$. brachypomus, $P$. pictus y G. morhua, lo cual implica movimientos peristálticos suaves y lentos en esta región del tubo digestivo (Morrison; Muñoz et al.; Olaya et al.).

Una característica importante encontrada en la mucosa y submucosa de $P$. axelrodi es el aumento de pliegues 
en forma de zig-zag o de malla (Smith), los cuales aumentan el área de contacto y mejoran la eficiencia de absorción de nutrientes (Vásquez; Albrecht et al.). Se observó un aumento de células caliciformes hacia la parte posterior del intestino, lo cual es un mecanismo de protección del epitelio y lubricación para permitir el paso de heces (Muñoz et al.).

En conclusión la conformación histológica del tracto digestivo de $P$. axelrodi presenta características propias de peces con hábitos alimenticios carnívoros, en este caso de presas pequeñas.

\section{AGRADECIMIENTOS}

A la Universidad Militar Nueva Granada, Vicerrectoría de Investigaciones, proyecto CIAS 2004-012.

GÓMEZ-RAMÍREZ, E.; OBANDO, M. J.; TOVAR, M. O.; CALDAS, M. L. \& HURTADO, H. Histological study of digestive tract of the Neón Cardenal Paracheirodon axelrodi (Characidae). Int. J. Morphol., 29(3):782-786, 2011.

SUMMARY: Cardinal neon, Paracheirodon axelrodi is the most exported ornamental fish in Colombia. However, many aspects of its biology are unknown. A histological study of the digestive tract was carried out and 15 specimens were sacrificed with MS 222, 0.5 $\mathrm{g} / \mathrm{L}$, fixed in $4 \%$ formaldehyde and decalcified with $7 \%$ nitric acid. H\&E technique was followed. P. axelrodi digestive tract showed four constitutive layers: mucosae, submucosae, muscular, and serosa. Mouth mucosae presents a stratified nonkeratinized squamous epithelium with many goblet cells, and no taste buds, submucosae with areolar connective tissue, and skeletal muscular layer. Teeth were conical and abundant. Along the whole intestinal tract the serous was a very thin connective tissue layer. Esophagus mucosae, submucosae, and muscular layer were similar to those of the mouth. However, muscular layer presented two layers in different orientations. Stomach mucosae were made of simple cylindrical epithelium with gastric glands. Submucosa did present areolar connective tissue, and muscular layer exhibited smooth muscle in two different orientations. The intestinal layers were similar to those of the stomach, but without gastric glands. Mucosae presented goblet cells especially at the posterior region. Intestinal folds were also observed. Tisular and morphological structure of $P$. axelrodi digestive tract suggests a small prey carnivorous fish.

KEY WORDS: Paracheirodon Axelrodi; Digestive tract; Histology; Morphology.

\section{REFERENCIAS BIBLIOGRÁFICAS}

Albrecht, M. P; Ferreira, M. F. \& Caramaschi, E. P. Anatomical features and histology of the digestive tract two related neotropical omnivorous fishes (Characiformes; Anostomidae). J. Fish Biol., 58(2):41930, 2000 .

Amin, A. B; Mortensen, L. \& Poppe, T. Histology atlas: normal structure of Salmonids. Bod, Norway, Akvapatologisk Laboratorium A. S., 1992. p.222.

Anderson, B. G. \& Mitchum, D. L. Atlas of trout histology. Wyoming, Fish and Game Department, 1974. p.110.

AVMA. Panel on Euthanasia. American Veterinary Medical Association. 2000 Report of the AVMA Panel on Euthanasia. J. Am. Vet. Med. Assoc., 218(5):669-96, 2001.

Beltrán, E. L. \& Beltrán, C. N. Contribución a la biología del bagre pintado Pseudoplatystoma fasciatum, Linnaeus, 1766 y su importancia pesquera. Tesis de pregrado (Biólogo marino). Universidad Jorge Tadeo Lozano, 1973. p.125.
Caceci, T; El-Habback, H. A; Smith, S. A \& Smith, B. J. The stomach of Oreochromis niloticus has three regions. J. Fish Biol., 50(5):939-52, 1997.

Collette, B.; Collette G. \& Facey, D. The diversity of fishes. Massachussets, Backwell Science editorial officers, 2000. p.528.

Jaramillo, J. A.; Gómez-Ramírez, E.; Caldas, M. L.; Rodríguez, D. \& Hurtado H. Histology and morphometry of dorsal root ganglia and their neurons in a fish of indeterminate growth the white cachama (Piaractus brachypomus). Actual Biol., 31:43-52, 2009.

Hepher, B. Nutrición de peces comerciales en estanques. Mexico D. F., Limusa S. A., 1993. p.406.

Mancera, N. J. \& Álvarez, R. Comercio de peces ornamentales en Colombia. Acta Biológica Colombiana., 13:23$52,2008$.

Morrison, C. Histology of the atlantic cod. Gadus morhua: An atlas, Digestive tract and associated organs. Ottawa, 
Canadian special publication of fisheries and aquatic sciences, 1987. p.177.

Muñoz, A.; Caldas, M. L. \& Hurtado, H. Análisis histomorfológico del sistema digestivo y glándulas anexas en alevinos de cachama blanca, Piaractus brachypomus (Characidae: Piaractus). Revista Facultad de Ciencias Básicas, 2:137-64, 2006.

Olaya, C. M.; Ovalle, C.; Gómez, E.; Rodríguez, D.; Caldas, M. L. \& Hurtado, H. Histología y morfométria del sistema digestivo del Silúrido bagre tigrito (Pimelodus pictus). Revista de la Facultad de Medicina Veterinaria y de Zootecnia, 54:311-23, 2007.

Saga, A. \& Román, C. Piscicultura: bases, métodos y aplicaciones. Armenia, AEBUQ, 1995. p.123.

Santana, C. \& Verdugo, P. Descripción histológica y anatómica del tracto digestivo del Nicuro (Pimelodus blochii, Valenciennens 1840) (Pises: Siluriformes, Pimelodidae). Tesis de pregrado (Biólogo), Universidad Militar Nueva Granada, 2003. p.75.

Steffens, W. Principios fundamentales de la alimentación de peces. Zaragoza, Acriba Ediciones, 1987. p.280.

Tovar, M. O.; Obando, M. J.; Gómez, E.; Caldas, M. L.; \& Hurtado, H. Histología y morfometría del ojo del pez dulceacuícola Paracheirodon axelrodi (Characiformes: Characidae). Int. J. Trop. Biol., 57(4):1107-18, 2009.

Vásquez, T. W. Principios de nutrición aplicada al cultivo de peces. Villavicencio, Universidad de los Llanos, 2004. p.101.

Walker, I. The food spectrum of the cardinal-tetra (Paracheirodon axelrodi, Characidae) in its natural habitat. Acta Amazonica, 34(1):69-73, 2004.
Dirección para correspondencia:

Edwin Gómez-Ramírez

Laboratorio de Histoembriología

Grupo de Ictiología, Programa de Biología Aplicada

Facultad de Ciencias

Universidad Militar Nueva Granada.

Bogotá

COLOMBIA

Email: edwin.gomez@unimilitar.edu.co

Recibido : 01-12-2010

Aceptado: 06-04-2011 